\title{
MOLECULAR CLOUD SPIRAL ARMS AND RESULTS FROM TIDAL INTERACTION MODELING
}

\author{
Ake Hja Imarson \\ Onsala Space Observatory, S-43900 Onsala, Sweden
}

I wish to report on some results from mapping of molecular cloud distributions in galaxies and from tidal interaction modeling - work performed at Onsala Space Observatory and in the Astrophysics Group of Institute of Theoretical Physics, Chalmers University of Technology/University of Goteborg.

There are a number of important questions in this context:

- Do molecular cloud spiral arms exist (and what is their location, width, structure)?

- Do interarm molecular clouds exist and at which mass fraction?

- What is the nature of the arm/interarm clouds (temperature, density, size, mass, velocity dispersion)?

- Where and how do dense clouds form?

- Do density waves, tidal interactions or bars play a role in the cloud/star formation processes, and how?

- Do molecular "superclouds" exist?

Detailed mapping of the CO(1-0) emissions of M51 and IC342 have become major projects for the Onsala $20 \mathrm{~m}$ telescope (beam width 33"), beginning with the first M51 observations in 1981.

M51. Spiral arm features, traced already in the raw 16" spacing data, became very conspicuous when the average radial brightness distribution was subtracted, as discussed already in Tokyo 1985 (Rydbeck etal. 1987). Radial streaming across arms in the sense predicted by density wave theory - is clearly observed. The estimated velocity shifts are surprisingly large, at least $50 \mathrm{~km} \mathrm{~s}^{-1}$ (Rydbeck etal. 1985, 1987).

A maximum entropy type deconvolution now has been applied to a more extensive data set (Rydbeck etal. 1988). While the antenna beam size is 33" the true resolution approaches $10^{\prime \prime}(0.5 \mathrm{kpc})$ where the signal is strong. The spiral arm distribution of molecular clouds then becomes very clear. The patchy structure of the arms suggests that the molecular clouds are assembled into giant complexes of mass up to $10^{8} M_{\infty}$. The average arm-interarm contrast is about 4 (actually this is a lower limit since it is hard to know how much of the signal left between the arms is due to error - and sidelobe contributions). With the higher angular resolution resulting from the MEM deconvolution large and abrupt tangential as well as radial velocity shift across arms are "observed". Spatial overlap of pre and post arm velocities is also evident. This suggests that cloud- cloud collisions must occur in the arms. 
IC342. The center of our CO map is dominated by the "bar" previously seen with the OVRO interferometer. However, connected to this bar spiral-arm-like features can also be traced (Wiklind etal. 1988). Arm-interarm contrasts of about 4 have been estimated for a few arm cuts. Like in M51 spatially overlapping pre and post. arm velocity componenets are observed. Although of much smaller magnitude than in M51 this velocity structure suggests cloud-cloud collisions in the spiral arms of IC 342 .

We note that for $25 \%$ of a disk in spiral phase an arm-interarm contrast of $3: 1$ (9:1) is expected if $50 \%(75 \%)$ of the $\mathrm{H}_{2}$ mass is contained in the arms. From these observations we may conclude that:

- Massive molecular cloud spiral arms do exist.

- Interarm molecular clouds may still contain a sizable mass fraction.

- Density waves (tidal interactions, bars) do play a role in organizing (existing) clouds into arms ("orbit crowding") and very massive cloud complexes. In M31 support has been found for formation of molecular clouds inside HI "superclouds" in a spiral arm region (Lada etal. 1988).

- Density waves (tidal interactions, bars) may also indirectly enhance high-mass star formation in the arms via observed cloud-cloud collisions. This suggestion is supported by the observed quadratic dependence of the number density of giant HII regions on the local $\mathrm{H}_{2}$ density in our Galaxy (Scoville etal. 1986).

Numerical simulations of tidal triggering of spiral arm structures in disk galaxies is a parallel development (cf Sundelius etal. 1987). Large, abrupt velocity shifts across arms and also "spatially overlapping pre and post arm velocity components" - like those observed in M51 - do indeed appear in simulations where impact parameters and perturber mass are similar to those of the M51 (NGC5194/5195) system. An ongoing detailed study of disk evolution vs time shows how tidal, material, arms first develop very suddenly - subsequently triggering density wave arms. This rapid organization of the gas into arms during the early phase of the tidal interaction presumably would lead to rather violent cloud- cloud collisions - and hence may be useful for our understanding of the IR/CO luminous "star-burst" galaxies.

\section{References:}

Lada, C.J., Margulis, M., Sofue, Y., Nakai, N. and Handa, T. 1988, Astrophys.J. $328,143$.

Rydbeck, G. Hjalmarson, A., and Rydbeck, O.E.H. 1985, Astron.Astrophys. 144, 282.

Rydbeck, G. Hjalmarson, A., Johansson, L.E.B., and Rydbeck, O.E.H. 1987, in Star Forming Regions, eds. Peimbert and Jugaku, Reidel, p.535.

Rydbeck, G., Hjalmarson, $\AA$., Wiklind, T., and Rydbeck, O.E.H. 1988, in Molecular Clouds in the Milky Way and Nearby Galaxies, eds. Dickman, Snell and Young, Springer.

Scoville, N.Z., Sanders, D.B., and Clemens, D.P. 1986, Astrophys.J. 310, L77.

Sundelius, B., Thomasson, M., Valtonen, M.J., and Byrd, G.G. 1987, Astron.Astrophys. 174, 67.

Wiklind, T., Rydbeck, G., Hjalmarson, $\AA$,, and Rydbeck, O.E.H. 1988, in Molecular clouds in the Milky Way and Nearby Galaxies, eds. Dickman, Snell and Young, Springer. 